\title{
Determination of the reference symmetry axis of a generally anisotropic medium which is approximately transversely isotropic
}

\author{
LUDĚK KLIMEŠ
}

Department of Geophysics, Faculty of Mathematics and Physics, Charles University in Prague, Ke Karlovu 3, 12116 Praha 2, Czech Republic (http://sw3d.cz/staff/klimes.htm)

Received: September 30, 2015; Revised: March 26, 2016; Accepted: April 18, 2016

\begin{abstract}
For a given stiffness tensor (tensor of elastic moduli) of a generally anisotropic medium, we estimate to what extent the medium is transversely isotropic (uniaxial) and determine the direction of its reference symmetry axis expressed in terms of the unit reference symmetry vector. If the medium is exactly transversely isotropic (exactly uniaxial), we obtain the direction of its symmetry axis. We can also calculate the first-order and second-order spatial derivatives of the reference symmetry vector which may be useful in tracing the reference rays for the coupling ray theory. The proposed method is tested using various transversely isotropic (uniaxial) and approximately transversely isotropic (approximately uniaxial) media.
\end{abstract}

Keywords: elastic anisotropy, stiffness tensor, elastic moduli, transverse isotropy, approximate transverse isotropy, reference symmetry axis

\section{INTRODUCTION}

The coupling ray theory (Coates and Chapman, 1990; Bulant and Kliměs, 2002; Klimeš and Bulant, 2012) is usually applied to common anisotropic rays (Bakker,

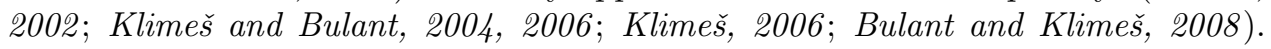
However, the coupling ray theory is more accurate if it is applied to reference rays which are closer to the actual S-wave paths (Klimeš and Bulant, 2014a, 2015).

If we know that a given medium is transversely isotropic (uniaxial), we can separate the slowness surface into the $\mathrm{P}$-wave slowness sheet, the $\mathrm{SH}$-wave slowness sheet and the SV-wave slowness sheet. We may then trace the $\mathrm{SH}$ rays and $\mathrm{SV}$ rays (Klimeš and Bulant, 2014a), and use them as the reference rays for the prevailingfrequency approximation of the coupling ray theory. In this case, the SH rays and $\mathrm{SV}$ rays are better reference rays than the common anisotropic reference rays.

Even if a given medium is not transversely isotropic but is approximately transversely isotropic (approximately uniaxial), the $\mathrm{SH}$ and $\mathrm{SV}$ reference rays (Klimers and Bulant, 2015) may represent better reference rays than the common anisotropic 


\section{Kliměs}

reference rays. Note that, in this case, the anisotropic-ray-theory rays often cannot be used as the reference rays (Bulant and Klimeš, 2014; Klimeš and Bulant, 2014b).

For a given stiffness tensor (tensor of elastic moduli) of a generally anisotropic medium, it is thus very useful to be able to estimate to what extent the medium is transversely isotropic and to determine the direction of its reference symmetry axis. This task is not only important for the accuracy of the coupling ray theory as explained above, but simultaneously represents a general problem of the nearest media approximation.

The stiffness tensor of a transversely isotropic medium is independent of the rotation around the symmetry axis.

For a given stiffness tensor of a generally anisotropic medium and a given rotation axis, we calculate the derivative of the stiffness tensor with respect to the angle of rotation in Section 2.1. The Frobenius norm of the derivative of the stiffness tensor with respect to the angle of rotation divided by the Frobenius norm of the stiffness tensor characterizes the strength of the dependence of the stiffness tensor on the rotation.

In Section 2.2, we determine the rotation axis by minimizing the Frobenius norm of the angular derivative of the stiffness tensor and refer to it as the reference symmetry axis. The direction of the reference symmetry axis is specified in terms of the reference symmetry vector. The stiffness tensor is axially rotated about a candidate reference symmetry axis, and the squared Frobenius norm of the derivative with respect to the rotation angle is expressed in terms of a quadratic form. The reference symmetry vector then appears as the eigenvector of this quadratic form associated with the minimum eigenvalue. We thus offer an analytically differentiable analytical solution of the problem which was initially solved by brute-force numerical methods (Norris, 2006; Moakher and Norris, 2006; Kochetov and Slawinski, 2008) or by very rough approximations (Arts et al., 1991).

In Sections 2.3 and 2.4, we also calculate the first-order and second-order spatial derivatives of the reference symmetry vector, which may be useful in tracing the SH and SV reference rays (Klimeš and Bulant, 2015) in heterogeneous velocity models with spatially varying reference symmetry vector, and for solving the corresponding equations of geodesic deviation (dynamic ray tracing equations).

The proposed method is tested in various transversely isotropic and approximately transversely isotropic media in Section 3.

The lower-case Roman indices take values 1, 2 and 3. The upper-case Roman indices take values 1 and 2 . Indices in parentheses are used to index the eigenvalues and corresponding eigenvectors. The Einstein summation over repetitive lower-case Roman indices (without parentheses) corresponding to the 3 spatial coordinates, is used throughout the paper. 
Reference symmetry axis of an approximately transversely isotropic medium

\section{REFERENCE SYMMETRY AXIS}

\subsection{Derivative of the stiffness tensor with respect to the angle of rotation}

Transformation matrix $R_{i n}\left(\varphi, t_{a}\right)$ corresponding to the rotation of vectors about a given unit vector $t_{a}$ by angle $\varphi$ is an orthogonal matrix, with $R_{i n}\left(0, t_{a}\right)=\delta_{i n}$, where Kronecker delta $\delta_{i n}$ represents the elements of the identity matrix. The derivative $\omega_{i n}=R_{i n}^{\prime}=\mathrm{d} R_{\text {in }} / \mathrm{d} \varphi$ of the transformation matrix at $\varphi=0$ reads

$$
\omega_{i n}\left(0, t_{a}\right)=-t_{m} \varepsilon_{\min },
$$

where $\varepsilon_{i j k}$ is the Levi-Civita symbol.

The rotated stiffness tensor reads

$$
a_{i j k l}\left(\varphi, t_{a}\right)=R_{i p}\left(\varphi, t_{a}\right) R_{j q}\left(\varphi, t_{b}\right) R_{k r}\left(\varphi, t_{c}\right) R_{l s}\left(\varphi, t_{d}\right) a_{p q r s},
$$

where $a_{\text {pqrs }}$ without arguments is the non-rotated tensor. Since rotation vector $t_{a}$ is unit, the derivative $a_{i j k l}^{\prime}=\mathrm{d} a_{i j k l} / \mathrm{d} \varphi\left(0, t_{a}\right)$ of stiffness tensor $a_{i j k l}\left(\varphi, t_{a}\right)$ with respect to the angle $\varphi$ of rotation at $\varphi=0$ follows directly from transformation (2),

$$
a_{i j k l}^{\prime}=\omega_{i n} a_{n j k l}+\omega_{j n} a_{i n k l}+\omega_{k n} a_{i j n l}+\omega_{l n} a_{i j k n} .
$$

We insert matrix (1) into angular derivative (3) and obtain

$$
a_{i j k l}^{\prime}=-t_{m}\left(\varepsilon_{m i n} a_{n j k l}+\varepsilon_{m j n} a_{i n k l}+\varepsilon_{m k n} a_{i j n l}+\varepsilon_{m l n} a_{i j k n}\right) .
$$

We define tensor

$$
d_{i j k l m}=\varepsilon_{m i n} a_{n j k l}+\varepsilon_{m j n} a_{i n k l}+\varepsilon_{m k n} a_{i j n l}+\varepsilon_{m l n} a_{i j k n}
$$

and express angular derivative (4) as

$$
a_{i j k l}^{\prime}=-d_{i j k l m} t_{m} .
$$

Note that if we put $a_{i j k l}^{\prime}=0$ here, we obtain the system of equations for the stiffness tensor of a transversely isotropic medium, equivalent to the equations of Cowin and Mehrabadi (1987).

\subsection{Reference symmetry vector}

We choose the square

$$
y=a_{i j k l}^{\prime} a_{i j k l}^{\prime}
$$

of the Frobenius norm of the derivative of the stiffness tensor with respect to the angle of rotation as the objective function. We insert derivative (6) into objective function (7) and obtain

$$
y=t_{m} B_{m n} t_{n},
$$

where

$$
B_{m n}=d_{i j k l m} d_{i j k l n} .
$$

The minimum value of objective function (8) over all unit vectors $t_{m}$ is attained for the eigenvector $t_{i(3)}$ of matrix $B_{m n}$ corresponding to the smallest eigenvalue $B_{(3)}$. 


\section{Kliměs}

We shall refer to this eigenvector $t_{i(3)}$ as the reference symmetry vector and to the corresponding direction as the reference symmetry axis.

The ratio

$$
\rho=\sqrt{\frac{a_{i j k l}^{\prime} a_{i j k l}^{\prime}}{a_{i j k l} a_{i j k l}}}
$$

of the Frobenius norm $\sqrt{a_{i j k l}^{\prime} a_{i j k l}^{\prime}}$ of the derivative of the stiffness tensor with respect to the angle of rotation and the Frobenius norm $\sqrt{a_{i j k l} a_{i j k l}}$ of the stiffness tensor characterizes how strongly the stiffness tensor depends on the rotation. For the reference symmetry vector $t_{i}=t_{i(3)}$, ratio (10) reads

$$
\rho_{(3)}=\sqrt{\frac{B_{(3)}}{a_{i j k l} a_{i j k l}}} .
$$

This ratio characterizes the extent to which the medium is not transversely isotropic. We shall thus refer to it as the non-TI ratio.

Note that the reference symmetry vector $t_{i(3)}$ is stable and has a good physical meaning only if the minimum eigenvalue $B_{(3)}$ of matrix $B_{m n}$ is considerably smaller than the two other eigenvalues $B_{(1)}$ and $B_{(2)}$, i.e. if $\rho_{(3)}$ is considerably smaller than ratios

$$
\rho_{(A)}=\sqrt{\frac{B_{(A)}}{a_{i j k l} a_{i j k l}}} .
$$

If non-TI ratio (11) is zero, the medium is exactly transversely isotropic and reference symmetry vector $t_{i(3)}$ specifies its symmetry axis.

\subsection{First-order spatial derivatives of the symmetry vector}

The symmetry vector may vary with spatial coordinates. If we use the spatially varying symmetry vector for tracing the $\mathrm{SH}$ and $\mathrm{SV}$ reference rays (Klimeš and Bulant, 2015), we need its first-order spatial derivatives.

Since the symmetry vector is a unit eigenvector of matrix (9), we can calculate its first-order and second-order partial derivatives with respect to spatial coordinates analogously to the derivatives of the eigenvectors of the Christoffel matrix.

In addition to reference symmetry vector $t_{i(3)}$ corresponding to the minimum eigenvalue $B_{(3)}$ of matrix (9), we introduce also the two other unit eigenvectors $t_{i(A)}$ corresponding to eigenvalues $B_{(A)}$.

The first-order partial derivatives of tensor (5) with respect to spatial coordinates read

$$
d_{i j k l m, p}=\varepsilon_{m i n} a_{n j k l, p}+\varepsilon_{m j n} a_{i n k l, p}+\varepsilon_{m k n} a_{i j n l, p}+\varepsilon_{m l n} a_{i j k n, p} .
$$

The first-order partial derivatives of matrix (9) with respect to spatial coordinates then read

$$
B_{m n, p}=d_{i j k l m, p} d_{i j k l n}+d_{i j k l m} d_{i j k l n, p}
$$


We transform the first-order partial derivatives of matrix (9) into eigenvectors $t_{i(a)}$,

$$
B_{(a b), p}=t_{m(a)} B_{m n, p} t_{n(b)} .
$$

Hereinafter, the subscripts in parentheses denote the covariant transform (15) into eigenvectors $t_{i(a)}$. The first-order partial derivatives of eigenvector $t_{i}=t_{i(3)}$ with respect to spatial coordinates then read (Kliměs, 2006, Eq. 17)

$$
t_{i, p}=\sum_{A} t_{i(A)} \frac{B_{(A 3), p}}{B_{(3)}-B_{(A)}} .
$$

2.4. Second-order spatial derivatives

of the symmetry vector

If we use the spatially varying symmetry vector for tracing the SH and SV reference rays (Kliměs and Bulant, 2015), we need its second--order spatial derivatives for calculating the geodesic deviation (dynamic ray tracing).

The second-order partial derivatives of tensor (5) with respect to spatial coordinates read

$$
d_{i j k l m, p q}=\varepsilon_{m i n} a_{n j k l, p q}+\varepsilon_{m j n} a_{i n k l, p q}+\varepsilon_{m k n} a_{i j n l, p q}+\varepsilon_{m l n} a_{i j k n, p q} .
$$

The second-order partial derivatives of matrix (9) with respect to spatial coordinates then read

$$
B_{m n, p q}=d_{i j k l m, p q} d_{i j k l n}+d_{i j k l m} d_{i j k l n, p q}+d_{i j k l m, p} d_{i j k l n, q}+d_{i j k l m, q} d_{i j k l n, p} .
$$

We transform the second-order partial derivatives of matrix (9) into eigenvectors $t_{i(A)}$,

$$
B_{(a b), p q}=t_{m(a)} B_{m n, p q} t_{n(b)} .
$$

The second-order partial derivatives of eigenvector $t_{i}=t_{i(3)}$ with respect to spatial coordinates then read (Klimeš and Bulant, 2015, Eqs 39-40)

$$
\begin{aligned}
t_{i, p q}=\sum_{A} t_{i(A)}\left(\frac{B_{(A 3), p q}}{B_{(3)}-B_{(A)}}\right. & -\frac{B_{(A 3), p} B_{(33), q}+B_{(A 3), q} B_{(33), p}}{\left(B_{(3)}-B_{(A)}\right)^{2}} \\
& \left.+\sum_{B} \frac{B_{(A B), p} B_{(B 3), q}+B_{(A B), q} B_{(B 3), p}}{\left(B_{(3)}-B_{(A)}\right)\left(B_{(3)}-B_{(B)}\right)}\right) \\
- & t_{i(3)} \sum_{B} \frac{B_{(B 3), p} B_{(B 3), q}}{\left(B_{(3)}-B_{(B)}\right)^{2}} .
\end{aligned}
$$




\section{Klimeš}

\section{NUMERICAL EXAMPLES}

Unit reference symmetry vector $t_{i(3)}$ and non-TI ratio (11) for a given stiffness tensor $a_{i j k l}$ is determined according to Sections 2.1 and 2.2 of this paper by new program tiaxis (Bucha and Bulant, 2015).

\subsection{Velocity model WA}

The density reduced stiffness tensor in the vertically heterogeneous $1-\mathrm{D}$ anisotropic velocity model WA by P̌̌enčı and Dellinger (2001) at the surface (zero depth) reads

$$
\left.\begin{array}{l}
11 \\
11 \\
22 \\
33 \\
23 \\
13 \\
12
\end{array}\right)\left(\begin{array}{cccccc}
13.39 & 4.46 & 4.46 & 0.00 & 0.00 & 0.00 \\
& 15.71 & 5.04 & 0.00 & 0.00 & 0.00 \\
& & 15.71 & 0.00 & 0.00 & 0.00 \\
& & & 5.33 & 0.00 & 0.00 \\
& & & & 4.98 & 0.00 \\
& & & & & 4.98
\end{array}\right) .
$$

Non-TI ratio (11) determined for this medium is

$$
\rho_{(3)}=0.000847,
$$

and the corresponding unit reference symmetry vector is

$$
t_{i(3)}=\left(\begin{array}{lll}
1.000000 & 0.000000 & 0.000000
\end{array}\right) .
$$

We see that the medium is not exactly transversely isotropic but is approximately transversely isotropic.

We now change density reduced stiffness tensor (21) slightly to density reduced stiffness tensor

$$
\begin{aligned}
& 11 \\
& 11 \\
& 22 \\
& 33 \\
& 23 \\
& 13 \\
& 12
\end{aligned}\left(\begin{array}{cccccc}
13.39 & 4.46 & 4.46 & 0.00 & 0.00 & 0.00 \\
& 15.70 & 5.04 & 0.00 & 0.00 & 0.00 \\
& & 15.70 & 0.00 & 0.00 & 0.00 \\
& & & 5.33 & 0.00 & 0.00 \\
& & & & 4.98 & 0.00 \\
& & & & & 4.98
\end{array}\right)
$$

of a transversely isotropic medium. Non-TI ratio (11) determined for this medium is

$$
\rho_{(3)}=0.000000
$$

and the corresponding unit reference symmetry vector is

$$
t_{i(3)}=\left(\begin{array}{lll}
1.000000 & 0.000000 & 0.000000
\end{array}\right) .
$$


Reference symmetry axis of an approximately transversely isotropic medium

\subsection{Velocity model QI}

Velocity model WA was rotated by $45^{\circ}$ about the positive $x_{3}$ half-axis in order to create vertically heterogeneous $1-\mathrm{D}$ anisotropic velocity model QI. The density reduced stiffness tensor in $\mathrm{km}^{2} \mathrm{~s}^{-2}$ in velocity model QI at the surface (zero depth) reads (Bulant and Kliměs, 2002, Eq. 38; Klimeš and Bulant, 2004, Eq. 57; Pšenčik et al., 2012, Eq. 16)

$\begin{aligned} & 11 \\ & 11 \\ & 22 \\ & 33 \\ & 23 \\ & 13 \\ & 12\end{aligned}\left(\begin{array}{rrrrrr}14.485 & 4.525 & 4.750 & 0.000 & 0.000 & -0.580 \\ & 14.485 & 4.750 & 0.000 & 0.000 & -0.580 \\ & & 15.710 & 0.000 & 0.000 & -0.290 \\ & & & 5.155 & -0.175 & 0.000 \\ & & & & 5.155 & 0.000 \\ & & & & & 5.045\end{array}\right)$.

Non-TI ratio (11) determined for this medium is

$$
\rho_{(3)}=0.000847,
$$

and the corresponding unit reference symmetry vector is

$$
t_{i(3)}=\left(\begin{array}{lll}
0.707107 & 0.707107 & 0.000000
\end{array}\right) .
$$

We see that the medium is not exactly transversely isotropic but is approximately transversely isotropic, analogously to velocity model WA.

\subsection{Velocity model KISS}

Velocity model WA was rotated by $1^{\circ}$ about the positive $x_{3}$ half-axis in order to create the vertically heterogeneous $1-\mathrm{D}$ anisotropic velocity model KISS. The density reduced stiffness tensor in $\mathrm{km}^{2} \mathrm{~s}^{-2}$ in velocity model KISS at the surface (zero depth) reads (P̌̌enčı et al., 2012, Eq. 20)

$\left.\begin{array}{l}11 \\ 11 \\ 22 \\ 33 \\ 23 \\ 13 \\ 12\end{array} \quad \begin{array}{rrrccc}13.39063 & 4.46008 & 4.46018 & 0.00000 & 0.00000 & -.01797 \\ & 15.70921 & 5.03982 & 0.00000 & 0.00000 & -.02251 \\ & & 15.71000 & 0.00000 & 0.00000 & -.01012 \\ & & & 5.32989 & -.00611 & 0.00000 \\ & & & & 4.98011 & 0.00000 \\ & & & & & 4.98008\end{array}\right)$.

Non-TI ratio (11) determined for this medium is

$$
\rho_{(3)}=0.000848,
$$

and the corresponding unit reference symmetry vector is

$$
t_{i(3)}=\left(\begin{array}{lll}
0.999848 & 0.017452 & 0.000000
\end{array}\right) .
$$

We see that the medium is not exactly transversely isotropic but is approximately transversely isotropic, analogously to velocity model WA. 


\section{Kliměs}

\subsection{Velocity model SC1_II}

The density reduced stiffness tensor in homogeneous anisotropic velocity model 1 by Shearer and Chapman (1989) reads

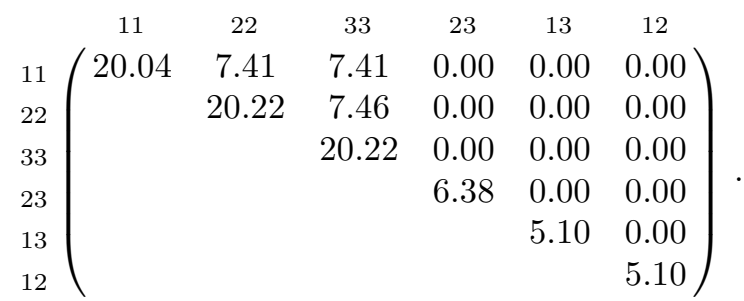

Non-TI ratio (11) determined for this medium is

$$
\rho_{(3)}=0.000000,
$$

and the corresponding unit reference symmetry vector is

$$
t_{i(3)}=\left(\begin{array}{lll}
1.000000 & 0.000000 & 0.000000
\end{array}\right) .
$$

We see that the medium is transversely isotropic within the rounding errors. If we inspect stiffness tensor (33) manually, we see that the medium is exactly transversely isotropic.

Velocity model 1 by Shearer and Chapman (1989) was first rotated by $45^{\circ}$ about the positive $x_{2}$ half-axis and then rotated by $30^{\circ}$ about the positive $x_{3}$ half-axis in order to create the stiffness tensor of the vertically heterogeneous $1-\mathrm{D}$ anisotropic velocity model SC1_II at the surface (zero depth). After these rotations, the symmetry vector should read

$$
t_{i(3)}=(\sqrt{3 / 8} \sqrt{1 / 8} \sqrt{1 / 2}) .
$$

The density reduced stiffness tensor in $\mathrm{km}^{2} \mathrm{~s}^{-2}$ in velocity model SC1_II at the surface (zero depth) reads (P̌̌enčik et al., 2012, Eq. 19)

$$
\begin{aligned}
& 11 \\
& 11 \\
& 22 \\
& 33 \\
& 23 \\
& 13 \\
& 12
\end{aligned}\left(\begin{array}{rrrrrr}
18.97125 & 7.67125 & 8.36125 & 0.46000 & -0.31177 & -0.15589 \\
& 19.64625 & 7.74375 & -0.49500 & 0.25115 & -0.42868 \\
& & 18.87000 & -0.02250 & -0.03897 & 0.53477 \\
& & & 5.89500 & 0.26847 & -0.28146 \\
& & & & 6.20500 & 0.15250 \\
& & & & & 5.97625
\end{array}\right) .
$$

Non-TI ratio (11) determined for this medium is

$$
\rho_{(3)}=0.000054
$$

and the corresponding unit reference symmetry vector is

$$
t_{i(3)}=\left(\begin{array}{lll}
0.612372 & 0.353554 & 0.707107
\end{array}\right) .
$$

We see that the medium is not exactly transversely isotropic but is close to transversely isotropic at the surface (zero depth). The difference from an exactly transversely isotropic medium is caused by the rounding errors in stiffness tensor (37). 
Numerically determined unit reference symmetry vector (39) is equal to its theoretical estimate (36).

At the depth of $1.5 \mathrm{~km}$, velocity model SC1_II is very close to isotropic, but is slightly cubic and its symmetry axes coincide with the coordinate axes. The density reduced stiffness tensor in $\mathrm{km}^{2} \mathrm{~s}^{-2}$ in velocity model SC1_II at the depth of $1.5 \mathrm{~km}$ reads (P̌̌enčık et al., 2012, Eq. 19)

$$
\begin{aligned}
& 11 \\
& 11 \\
& 22 \\
& 33 \\
& 23 \\
& 13 \\
& 12
\end{aligned}\left(\begin{array}{cccccc}
30.25 & 10.08 & 10.08 & 0.00 & 0.00 & 0.00 \\
& 30.25 & 10.08 & 0.00 & 0.00 & 0.00 \\
& & 30.25 & 0.00 & 0.00 & 0.00 \\
& & & 10.08 & 0.00 & 0.00 \\
& & & & 10.08 & 0.00 \\
& & & & & 10.08
\end{array}\right) .
$$

The elements of the stiffness tensor (elastic moduli) are linear functions of depth. This means that, at depths between $0 \mathrm{~km}$ and $1.5 \mathrm{~km}$, velocity model SC1_II is close to transversely isotropic, but is slightly tetragonal. For example, at the depth of $1.4 \mathrm{~km}$, non-TI ratio (11) is

$$
\rho_{(3)}=0.000397,
$$

and the corresponding unit reference symmetry vector is

$$
t_{i(3)}=\left(\begin{array}{lll}
0.611611 & 0.348810 & 0.710115
\end{array}\right) .
$$

We see that the medium is less transversely isotropic at the depth of $1.4 \mathrm{~km}$ than at the surface.

\subsection{Orthorhombic medium}

The orthorhombic medium by Schoenberg and Helbig (1997) was already used by Bucha (2013c, 2014a). The density reduced stiffness tensor reads

$\begin{aligned} & 11 \\ & 11 \\ & 22 \\ & 33 \\ & 23 \\ & 13 \\ & 12\end{aligned}\left(\begin{array}{cccccc}9.0000 & 3.6000 & 2.2500 & 0.0000 & 0.0000 & 0.0000 \\ & 9.8400 & 2.4000 & 0.0000 & 0.0000 & 0.0000 \\ & & 5.9375 & 0.0000 & 0.0000 & 0.0000 \\ & & & 2.0000 & 0.0000 & 0.0000 \\ & & & & 1.6000 & 0.0000 \\ & & & & & 2.1820\end{array}\right)$.

Non-TI ratio (11) determined for this medium is

$$
\rho_{(3)}=0.254022,
$$

and the corresponding unit reference symmetry vector is

$$
t_{i(3)}=\left(\begin{array}{lll}
0.000000 & 0.000000 & 1.000000
\end{array}\right) .
$$

Ratios (12) read

$$
\rho_{(2)}=0.433103, \quad \rho_{(1)}=0.436127 .
$$

We see that this orthorhombic medium contains a considerable transversely isotropic component with the vertical axis of symmetry. 


\section{Kliměs}

\subsection{Triclinic medium}

The triclinic medium by Mensch and Rasolofosaon (1997) was already used by Bucha (2012, 2013a,b,c, 2014b, 2015, 2016). The density reduced stiffness tensor reads

$$
\begin{aligned}
& 11 \\
& 11 \\
& 22 \\
& 33 \\
& 23 \\
& 13 \\
& 12
\end{aligned}\left(\begin{array}{cccccc}
10.3 & 0.9 & 1.3 & 1.4 & 1.1 & 0.8 \\
& 10.6 & 2.1 & 0.2 & -0.2 & -0.6 \\
& & 14.1 & 0.0 & -0.5 & -1.0 \\
& & & 5.1 & 0.0 & 0.2 \\
& & & & 6.0 & 0.0 \\
& & & & & 4.9
\end{array}\right) .
$$

Non-TI ratio (11) determined for this medium is

$$
\rho_{(3)}=0.275710
$$

and the corresponding unit reference symmetry vector is

$$
t_{i(3)}=\left(\begin{array}{lll}
0.576529 & 0.466767 & 0.670629
\end{array}\right) .
$$

Ratios (12) read

$$
\rho_{(2)}=0.353717, \quad \rho_{(1)}=0.545104 .
$$

We see that this triclinic medium contains a considerable transversely isotropic component with a tilted axis of symmetry.

\section{APPLICATIONS}

The possibility of determining whether a given stiffness tensor corresponds to a transversely isotropic medium may be very useful in selecting the method for calculating the wave field. If the medium is transversely isotropic or approximately transversely isotropic, we may use its symmetry vector or reference symmetry vector in tracing the $\mathrm{SH}$ and $\mathrm{SV}$ rays or the $\mathrm{SH}$ and $\mathrm{SV}$ reference rays (Klimeš and Bulant, 2015). The non-TI ratio, which identifies how much the given medium is transversely isotropic, and the unit reference symmetry vector can be determined according to Sections 2.1 and 2.2 of this paper.

If the reference symmetry vector is spatially varying, we also need its first-order spatial derivatives for ray tracing, and its second-order spatial derivatives for solving the corresponding equations of geodesic deviation (dynamic ray tracing equations). The first-order spatial derivatives of the reference symmetry vector can be determined according to Section 2.3 of this paper. The second-order spatial derivatives of the reference symmetry vector can be determined according to Section 2.4 of this paper. 


\section{Reference symmetry axis of an approximately transversely isotropic medium}

Acknowledgements: The suggestions by Peter Bakker and Yuriy Ivanov made it possible for me to improve the paper considerably. The research has been supported by the Grant Agency of the Czech Republic under contracts 16-01312S and 16-05237S, by the Ministry of Education, Youth and Sports of the Czech Republic within research project CzechGeo/EPOS LM2015079, and by the members of the consortium "Seismic Waves in Complex 3-D Structures" (see "http://sw3d.cz").

\section{References}

Arts R.J., Helbig K. and Rasolofosaon P.N.J., 1991. General anisotropic elastic tensor in rocks: approximation, invariants, and particular directions. SEG Technical Program Expanded Abstracts 1991, 10, 1534-1537.

Bakker P.M., 2002. Coupled anisotropic shear wave raytracing in situations where associated slowness sheets are almost tangent. Pure Appl. Geophys., 159, 1403-1417.

Bucha V., 2012. Kirchhoff prestack depth migration in 3-D simple models: comparison of triclinic anisotropy with simpler anisotropies. Stud. Geophys. Geod., 56, 533-552.

Bucha V., 2013a. Kirchhoff prestack depth migration in velocity models with and without vertical gradients: Comparison of triclinic anisotropy with simpler anisotropies. Seismic Waves in Complex 3-D Structures, 23, 45-59 (http://sw3d.cz).

Bucha V., 2013b. Kirchhoff prestack depth migration in velocity models with and without rotation of the tensor of elastic moduli: Poorly displayed part of migrated interface in correct model with triclinic anisotropy. Seismic Waves in Complex 3-D Structures, 23, 61-69 (http://sw3d.cz).

Bucha V., 2013c. Kirchhoff prestack depth migration in velocity models with and without rotation of the tensor of elastic moduli: Orthorhombic and triclinic anisotropy. Seismic Waves in Complex 3-D Structures, 23, 71-80 (http://sw3d.cz).

Bucha V., 2014a. Kirchhoff prestack depth migration in orthorhombic velocity models with differently rotated tensors of elastic moduli. Seismic Waves in Complex 3-D Structures, 24, 59-75 (http://sw3d.cz).

Bucha V., 2014b. Kirchhoff prestack depth migration in triclinic velocity models with differently rotated tensors of elastic moduli. Seismic Waves in Complex 3-D Structures, 24, 77-93 (http://sw3d.cz).

Bucha V., 2015. Kirchhoff prestack depth migration in a homogeneous triclinic velocity model for P, S and converted waves. Seismic Waves in Complex 3-D Structures, 25, 89-102 (http://sw3d.cz).

Bucha V., 2016. Kirchhoff prestack depth migration in simple orthorhombic and triclinic models with differently rotated elasticity tensor: comparison with zero-offset traveltime perturbations. Seismic Waves in Complex 3-D Structures, 26, 81-102 (http:/ /sw3d.cz).

Bucha V. and Bulant P. (Eds), 2015. SW3D-CD-19 (DVD-ROM). Seismic Waves in Complex 3-D Structures, 25, 209-210 (http://sw3d.cz).

Bulant P. and Klimeš L., 2002. Numerical algorithm of the coupling ray theory in weakly anisotropic media. Pure Appl. Geophys., 159, 1419-1435.

Bulant P. and Klimeš L., 2008. Numerical comparison of the isotropic-common-ray and anisotropic-common-ray approximations of the coupling ray theory. Geophys. J. Int., 175, 357-374. 


\section{Kliměs}

Bulant P. and Klimeš L., 2014. Anisotropic-ray-theory geodesic deviation and two-point ray tracing through a split intersection singularity. Seismic Waves in Complex 3-D Structures, 24, 179-187 (http://sw3d.cz).

Coates R.T. and Chapman C.H., 1990. Quasi-shear wave coupling in weakly anisotropic 3-D media. Geophys. J. Int., 103, 301-320.

Cowin S.C. and Mehrabadi M.M., 1987. On the identification of material symmetry for anisotropic elastic materials. Quart. J. Mech. Appl. Math., 40, 451-476.

Klimeš L., 2006. Common-ray tracing and dynamic ray tracing for $\mathrm{S}$ waves in a smooth elastic anisotropic medium. Stud. Geophys. Geod., 50, 449-461.

Klimeš L. and Bulant P., 2004. Errors due to the common ray approximations of the coupling ray theory. Stud. Geophys. Geod., 48, 117-142.

Klimeš L. and Bulant P., 2006. Errors due to the anisotropic-common-ray approximation of the coupling ray theory. Stud. Geophys. Geod., 50, 463-477.

Klimeš L. and Bulant P., 2012. Single-frequency approximation of the coupling ray theory. Seismic Waves in Complex 3-D Structures, 22, 143-167 (http://sw3d.cz).

Klimeš L. and Bulant P., 2014a. Prevailing-frequency approximation of the coupling ray theory for $\mathrm{S}$ waves along the $\mathrm{SH}$ and $\mathrm{SV}$ reference rays in a transversely isotropic medium. Seismic Waves in Complex 3-D Structures, 24, 165-177 (http://sw3d.cz).

Klimeš L. and Bulant P., 2014b. Anisotropic-ray-theory rays in velocity model SC1-II with a split intersection singularity. Seismic Waves in Complex 3-D Structures, 24, 189-205 (http://sw3d.cz).

Klimeš L. and Bulant P., 2015. Ray tracing and geodesic deviation of the SH and SV reference rays in a heterogeneous generally anisotropic medium which is approximately transversely isotropic. Seismic Waves in Complex 3-D Structures, 25, 187-208 (http:/ /sw3d.cz).

Kochetov M. and Slawinski M.A., 2008. On obtaining effective transversely isotropic elasticity tensors. J. Elast., 94, 1-13.

Mensch T. and Rasolofosaon P., 1997. Elastic-wave velocities in anisotropic media of arbitrary symmetry-generalization of Thomsen's parameters $\epsilon, \delta$ and $\gamma$. Geophys. J. Int., 128, 43-64.

Moakher M. and Norris A.N., 2006. The closest elastic tensor of arbitrary symmetry to an elasticity tensor of lower symmetry. J. Elast., 85, 215-263.

Norris A.N., 2006. Elastic moduli approximation of higher symmetry for the acoustical properties of an anisotropic material. J. Acoust. Soc. Am., 119, 2114-2121.

Pšenčík I. and Dellinger J., 2001. Quasi-shear waves in inhomogeneous weakly anisotropic media by the quasi-isotropic approach: A model study. Geophysics, 66, 308-319.

Pšenčík I., Farra V. and Tessmer E., 2012. Comparison of the FORT approximation of the coupling ray theory with the Fourier pseudospectral method. Stud. Geophys. Geod., 56, 35-64.

Schoenberg M. and Helbig K., 1997. Orthorhombic media: Modeling elastic wave behavior in a vertically fractured earth. Geophysics, 62, 1954-1974.

Shearer P.M. and Chapman C.H., 1989. Ray tracing in azimuthally anisotropic media I. Results for models of aligned cracks in the upper crust. Geophys. J., 96, 51-64. 